PROCEEDINGS OF THE

AMERICAN MATHEMATICAL SOCIETY

Volume 132, Number 8, Pages 2331-2338

S 0002-9939(04)07358-7

Article electronically published on February 19, 2004

\title{
A STRONG LEBESGUE POINT PROPERTY FOR SOBOLEV FUNCTIONS
}

\author{
VISA LATVALA
}

(Communicated by Juha M. Heinonen)

\begin{abstract}
We show that first-order Sobolev functions fulfill a Wiener integral type Lebesgue point property outside a set of Sobolev capacity zero. Our condition is stronger than the standard Lebesgue point property, but the exceptional set is slightly larger.
\end{abstract}

\section{INTRODUCTION}

The classical Lebesgue differentiation theorem in $\mathbf{R}^{n}$ asserts that almost every point $x_{0}$ of a locally integrable function $u$ is a Lebesgue point, i.e., it satisfies

$$
\lim _{r \rightarrow 0} \frac{1}{\left|B\left(x_{0}, r\right)\right|} \int_{B\left(x_{0}, r\right)}\left|u-u\left(x_{0}\right)\right| d x=0 .
$$

For Sobolev functions the condition (1.1) holds with a higher integrability exponent outside a smaller exceptional set. The following result is by now well known and widely used: Let $1<p \leq n$ and let $u$ be a locally $L^{p}$-integrable function whose distributional partial derivatives are locally $L^{p}$-integrable. Then

$$
\lim _{r \rightarrow 0} \frac{1}{\left|B\left(x_{0}, r\right)\right|} \int_{B\left(x_{0}, r\right)}\left|u-u\left(x_{0}\right)\right|^{s} d x=0
$$

for all $x_{0} \in \mathbf{R}^{n}$ outside a set of $p$-capacity zero whenever $s>0$ is a finite exponent not greater than the Sobolev conjugate of $p$. This result appeared first in [5] and [14. Nowadays the proof can be found in standard textbooks on Sobolev functions; see e.g. [4] or [15].

As the main result of this paper we improve the condition (1.2) by showing that the integral averages in (1.2) fulfill the following Wiener integral type condition:

Theorem 1.1. Let $u \in W^{1, p}\left(\mathbf{R}^{n}\right)$ be p-quasicontinuous, and let $1<q<p$. If $1<p<n$, then for q-q.e. $x_{0} \in \mathbf{R}^{n}$ and for all $0<s \leq \frac{n p}{n-p}$,

$$
\int_{0}^{1}\left(\frac{1}{\left|B\left(x_{0}, r\right)\right|} \int_{B\left(x_{0}, r\right)}\left|u-u\left(x_{0}\right)\right|^{s} d x\right)^{\frac{p}{s(p-1)}} \frac{d r}{r}<\infty .
$$

Received by the editors January 23, 2003 and, in revised form, April 29, 2003.

2000 Mathematics Subject Classification. Primary 46E35; Secondary 31C15.

Key words and phrases. Sobolev functions, Lebesgue points, capacity. 


$$
\begin{aligned}
& \text { If } p=n \text {, then for } q \text {-q.e. } x_{0} \in \mathbf{R}^{n} \text { and for all } s>0 \text {, } \\
& \qquad \int_{0}^{1}\left(\frac{1}{\left|B\left(x_{0}, r\right)\right|} \int_{B\left(x_{0}, r\right)}\left|u-u\left(x_{0}\right)\right|^{s} d x\right)^{\frac{p}{s(p-1)}} \frac{d r}{r}<\infty .
\end{aligned}
$$

Notice that Sobolev functions are locally Hölder continuous if $p>n$ (see [4], p. 143). In this case our theorem trivially holds for all $x_{0} \in \mathbf{R}^{n}$. This is why we assume above that $1<p \leq n$. We explain in Remark 3.1 why the convergence of the Wiener type integrals in Theorem 1.1 is a stronger condition than the condition (1.2). Notice that the property (1.2) holds outside a set of Sobolev $p$-capacity zero. Hence the exceptional set for Theorem 1.1 is larger than the exceptional set for (1.2). On the other hand, by choosing a sequence $1<q_{i}<p$ with $\lim _{i \rightarrow \infty} q_{i}=p$ we easily obtain from well-known results on Hausdorff measure and capacity that the Hausdorff dimension of the exceptional set in Theorem 1.1 is at most $n-p$ (see e.g. [4] or [15]).

This paper is organized as follows. In section 2 we briefly recall the necessary concepts and known results that are necessary for understanding the proof of our main result. In section 3 we prove Theorem 1.1 and make some supplementary comments. The new feature in our proof is that we use fine topological arguments in order to be able to use a version of the Sobolev-Poincaré inequality that fits into our considerations.

\section{BACKGROUND}

We denote by $|E|$ the $n$-dimensional Lebesgue measure of the set $E \subset \mathbf{R}^{n}, n \geq 2$. In integrals $d x$ (resp. $d r$ ) refers to integration with respect to $n$-dimensional (resp. 1-dimensional) Lebesgue measure.

Let $\Omega \subset \mathbf{R}^{n}$ be an open set, and let $p \geq 1$. The Sobolev space $W^{1, p}(\Omega)$ consists of functions $u \in L^{p}(\Omega)$ for which $|\nabla u| \in L^{p}(\Omega)$. Here and elsewhere, $\nabla u$ is the distributional gradient of $u$.

The first auxiliary lemma is an easy consequence of the Sobolev-Poincaré inequality. Since its proof is not easily found in the literature, we give a proof here. Our proof is the same as in [9], Lemma 2.1, where the authors however study Sobolev functions on metric spaces.

Lemma 2.1. Let $u \in W^{1, p}(B)$, where $1<p<n$ and $B=B\left(x_{0}, r\right)$. Suppose that

$$
|\{x \in B:|u(x)|>0\}| \leq \kappa|B|
$$

for some $0<\kappa<1$, and let $1 \leq s \leq \frac{n p}{n-p}$. Then there is a constant $c$, depending only on $n, p, s$ and $\kappa$, such that

$$
\left(\frac{1}{|B|} \int_{B}|u|^{s} d x\right)^{\frac{1}{s}} \leq c r\left(\frac{1}{|B|} \int_{B}|\nabla u|^{p} d x\right)^{\frac{1}{p}} .
$$

Proof. By the Minkowski inequality,

$$
\left(\int_{B}|u|^{s} d x\right)^{\frac{1}{s}} \leq\left(\int_{B}\left|u-u_{B}\right|^{s} d x\right)^{\frac{1}{s}}+\left|u_{B}\right||B|^{\frac{1}{s}},
$$


where $u_{B}$ denotes the integral average $u_{B}=\frac{1}{|B|} \int_{B} u d x$. By the Sobolev-Poincaré inequality ([15], Corollary 1.64),

$$
\left(\int_{B}\left|u-u_{B}\right|^{s} d x\right)^{\frac{1}{s}} \leq c|B|^{\frac{1}{s}-\frac{1}{p}} r\left(\int_{B}|\nabla u|^{p} d x\right)^{\frac{1}{p}}
$$

where $c$ depends only on $n, p$ and $s$. By the Hölder inequality,

$$
\begin{aligned}
\left|u_{B}\right||B|^{\frac{1}{s}} & \leq|B|^{\frac{1}{s}-1} \int_{\{x \in B|u(x)|>0\}}|u| d x \\
& \leq\left(\int_{B}|u|^{s} d x\right)^{\frac{1}{s}}\left(\frac{|\{x \in B|u(x)|>0\}|}{|B|}\right)^{1-\frac{1}{s}} \leq\left(\int_{B}|u|^{s} d x\right)^{\frac{1}{s}} \kappa^{1-\frac{1}{s}} .
\end{aligned}
$$

The assertion follows by combining the inequalities.

2.1. Capacity. We need two different capacities. Let $p>1$, and let $E \subset \mathbf{R}^{n}$. The Sobolev p-capacity of $E$ is defined by

$$
C_{p}(E)=\inf _{u} \int_{\mathbf{R}^{n}}\left(|u|^{p}+|\nabla u|^{p}\right) d x
$$

where the infimum is taken over all $u \in W^{1, p}\left(\mathbf{R}^{n}\right)$ such that $u \geq 1$ on an open neighbourhood of $E$. The Sobolev $p$-capacity is studied in many books (see e.g. [4], [8], [15]). The Bessel $(1, p)$-capacity of $E$ is defined as

$$
B_{1, p}(E)=\inf \left\{\|f\|_{p}^{p}: f \in L_{+}^{p}\left(\mathbf{R}^{n}\right), g_{1} * f \geq 1 \text { in } E\right\} .
$$

We refer to [1] and [16] for the properties of the Bessel kernel $g_{1}$ and the Bessel capacity $B_{1, p}$. We only need the fact that $g_{1}(r)$ behaves like $r^{1-n}$ as $r \rightarrow 0^{+}$([1], p. 11). It is known that the capacities $C_{p}$ and $B_{1, p}$ are equivalent; see e.g. [10], Remark 1.13, i.e., there is a constant $c>0$, depending only on $n$ and $p$, such that

$$
c^{-1} C_{p}(E) \leq B_{1, p}(E) \leq c C_{p}(E)
$$

for all $E \subset \mathbf{R}^{n}$. This implies that all concepts we relate to the capacity are the same for $C_{p}$ and $B_{1, p}$.

We next introduce the notions of $p$-quasicontinuity and $p$-fine continuity. These closely related concepts are the key tools for understanding the precise behaviour of Sobolev functions. A function $f: \Omega \rightarrow[-\infty,+\infty]$ is p-quasicontinuous in $\Omega$ if for each $\varepsilon>0$ there is an open set $G$ such that $C_{p}(G)<\varepsilon$ and $f_{\mid \Omega \backslash G}$ is finite and continuous. For $p \leq n$, there are Sobolev functions that do not have a continuous representative. The lack of continuity can be partially compensated by the fact that any function $u \in W^{1, p}\left(\mathbf{R}^{n}\right)$ has a $p$-quasicontinuous representative ([15], Theorem 2.20). It is even possible to show that any function $u \in W^{1, p}\left(\mathbf{R}^{n}\right)$ has a representative that is Hölder continuous outside a set of arbitrary small $q$-capacity, $1<q<p$. This is the content of the second auxiliary lemma.

Lemma 2.2. Let $1<p \leq n$, and let $u \in W^{1, p}\left(\mathbf{R}^{n}\right)$ be $p$-quasicontinuous. Then for each $\varepsilon>0$ and for each $1<q<p$ there are $E \subset \mathbf{R}^{n}$ and constants $\alpha>0$, $C_{0}>0$ such that $C_{q}(E)<\varepsilon$ and

$$
|u(x)-u(y)| \leq C_{0}|x-y|^{\alpha}
$$

for all $x, y \in \mathbf{R}^{n} \backslash E$. 
Lemma 2.2 was first proved in [12], Theorem 7. A different, more general proof is given in [6].

In order to define the $p$-fine topology, we first recall the concept of $p$-thinness. A set $E \subset \mathbf{R}^{n}$ is called $p$-thin at $x_{0} \in \mathbf{R}^{n}$ if

$$
\int_{0}^{1}\left(\frac{C_{p}\left(E \cap B\left(x_{0}, r\right)\right)}{r^{n-p}}\right)^{\frac{1}{p-1}} \frac{d r}{r}<\infty .
$$

The integral in (2.1) is called the Wiener integral. The Wiener integral is the key tool for characterizing the regular boundary points of the Dirichlet problem for the $p$-Laplacian; see [8] and [11]. A point $x \in U \subset \mathbf{R}^{n}$ is called a $p$-fine interior point of $U$ if $\mathbf{R}^{n} \backslash U$ is $p$-thin at $x$. This definition gives rise to a topology called the $p$-fine topology. We denote by $\bar{E}^{p}$ the $p$-fine closure of $E$. The role of the $p$-fine topology for nonlinear elliptic PDEs is carefully studied in [15]. The next lemma is proved in [15], Theorem 2.140 (the original proof goes back to [3] and [7]; see [2], Proposition 2).

Lemma 2.3. Let $p>1$, and let $E \subset \mathbf{R}^{n}$. Then

$$
C_{p}(E)=C_{p}\left(\bar{E}^{p}\right) .
$$

Our ideas essentially rely on the following result, due to Meyers. If $1<p<n$ and $u \in W^{1, p}\left(\mathbf{R}^{n}\right)$, then by [13], Theorem 2.1,

$$
\int_{0}^{\infty}\left(g_{1}^{p}(4 r) r^{n(p-1)} \int_{B\left(x_{0}, r\right)}|\nabla u|^{p} d x\right)^{\frac{1}{p-1}} \frac{d r}{r}<\infty \text { for } p \text {-q.e. } x_{0} \text { in } \mathbf{R}^{n} .
$$

Here and elsewhere we use the phrase $p$-q.e. to express that some pointwise property holds outside a set of Sobolev $p$-capacity zero. Since the Bessel kernel $g_{1}(r)$ is comparable to $r^{1-n}$ as $r \rightarrow 0^{+}$, we obtain the following lemma:

Lemma 2.4. Let $1<p<n$, and let $u \in W^{1, p}\left(\mathbf{R}^{n}\right)$. Then

$$
\int_{0}^{1}\left(r^{p-n} \int_{B\left(x_{0}, r\right)}|\nabla u|^{p} d x\right)^{\frac{1}{p-1}} \frac{d r}{r}<\infty \quad \text { for } p \text {-q.e. } x_{0} \text { in } \mathbf{R}^{n} .
$$

Lemma 2.4 can be used to show that any Sobolev function $u \in W^{1, p}\left(\mathbf{R}^{n}\right)$ has a representative that is $p$-finely continuous outside a set $E$ with $C_{p}(E)=0([16$, pp. 124-125). This is much more difficult to prove than the existence of a $p$ quasicontinuous representative.

\section{The PRoOf of Theorem 1.1}

For the reader's convenience we first briefly discuss the key idea behind the improvements of the Lebesgue theorem for Sobolev functions; see 4], pp. 160-162, or [15], pp. 90-92.

Let $x_{0} \in \mathbf{R}^{n}$ be a Lebesgue point of a function $u \in W^{1, p}\left(\mathbf{R}^{n}\right)$. By the Minkowski inequality,

$$
\begin{aligned}
& \left(\frac{1}{\left|B\left(x_{0}, r\right)\right|} \int_{B\left(x_{0}, r\right)}\left|u-u\left(x_{0}\right)\right|^{p} d x\right)^{\frac{1}{p}} \\
\leq & \left(\frac{1}{\left|B\left(x_{0}, r\right)\right|} \int_{B\left(x_{0}, r\right)}\left|u-u_{B\left(x_{0}, r\right)}\right|^{p} d x\right)^{\frac{1}{p}}+\left|u\left(x_{0}\right)-u_{B\left(x_{0}, r\right)}\right|,
\end{aligned}
$$


and by the Sobolev-Poincaré inequality,

$$
\left(\frac{1}{\left|B\left(x_{0}, r\right)\right|} \int_{B\left(x_{0}, r\right)}\left|u-u_{B\left(x_{0}, r\right)}\right|^{p} d x\right)^{\frac{1}{p}} \leq c r^{p-n} \int_{B\left(x_{0}, r\right)}|\nabla u|^{p} d x .
$$

By [15], Corollary 1.19, $\lim _{r \rightarrow 0} r^{p-n} \int_{B\left(x_{0}, r\right)}|\nabla u|^{p} d x=0$ for $p$-q.e. $x_{0}$. Hence (1.2) will follow once it is shown that $p$-q.e. $x_{0}$ in $\mathbf{R}^{n}$ is a Lebesgue point.

Why doesn't the estimate (3.1) yield the proof of Theorem 1.1? Lemma 2.4 guarantees that the first term on the right-hand side of (3.1) does not cause any harm, but there is no easy way to deal with the second term. To overcome this difficulty, instead of writing the estimate (3.1) we divide the set $B\left(x_{0}, r\right)$ into two parts. Because of the quasi Hölder continuity of Lemma 2.2 the "good" part does not cause any harm, and the fine topological arguments guarantee that we are able to use Lemma 2.1 to deal with the "bad" part.

Proof of Theorem 1.1. Notice that $u\left(x_{0}\right)$ is finite $p$-q.e. in $\mathbf{R}^{n}$, since $u$ is $p$-quasicontinuous. Assume first that $1<p<n, 0<s \leq \frac{n p}{n-p}$, and put $v(x)=\left|u(x)-u\left(x_{0}\right)\right|$. By Lemma 2.2, we are able to choose $E_{i} \subset \mathbf{R}^{n}$ such that $C_{q}\left(E_{i}\right)<\frac{1}{i}$ and $u$ is $\alpha$-Hölder continuous in $\mathbf{R}^{n} \backslash E_{i}$. Set

$$
E=\left\{x \in \mathbf{R}^{n}: \int_{0}^{1}\left(r^{p-n} \int_{B\left(x_{0}, r\right)}|\nabla u|^{p} d x\right)^{\frac{1}{p-1}} \frac{d r}{r}=\infty\right\} \cup \bigcap_{i=1}^{\infty} \bar{E}_{i}^{q},
$$

where $\bar{E}_{i}^{q}$ denotes the $q$-fine closure of $E_{i}$. Since the sets of $p$-capacity zero are of $q$ capacity zero (15, Lemma 2.14), we infer that $C_{q}(E)=0$ by using Lemmas 2.3 and 2.4 together with the fact that the capacity is a monotone countably subadditive set function. Let $x_{0} \notin E$ and choose an index $i$ such that $x_{0} \notin \bar{E}_{i}^{q}$. Then there are constants $C_{0}>0$ and $\alpha>0$ such that

$$
\left|u(x)-u\left(x_{0}\right)\right| \leq C_{0}\left|x-x_{0}\right|^{\alpha}
$$

for all $x \in \mathbf{R}^{n} \backslash \bar{E}_{i}^{q}$. Let $C_{0}>0$ and $\alpha>0$ be the constants satisfying (3.2), and define $U=\mathbf{R}^{n} \backslash \bar{E}_{i}^{q}, B_{r}=B\left(x_{0}, r\right)$. We divide our integral into two parts by writing

$$
\left(\frac{1}{\left|B_{r}\right|} \int_{B_{r}} v^{s} d x\right)^{\frac{p}{s}} \leq 2^{\frac{p}{s}}\left(\frac{1}{\left|B_{r}\right|} \int_{B_{r} \cap U} v^{s} d x\right)^{\frac{p}{s}}+2^{\frac{p}{s}}\left(\frac{1}{\left|B_{r}\right|} \int_{B_{r} \backslash U} v^{s} d x\right)^{\frac{p}{s}} .
$$

The first term on the right-hand side is trivially estimated as

$$
\left(\frac{1}{\left|B_{r}\right|} \int_{B_{r} \cap U} v^{s} d x\right)^{\frac{p}{s}} \leq C_{0}^{p} r^{\alpha p}
$$


To estimate the second term, observe that

$$
\begin{aligned}
\left(\frac{1}{\left|B_{r}\right|} \int_{B_{r} \backslash U} v^{s} d x\right)^{\frac{p}{s}} & \leq\left(\frac{1}{\left|B_{r}\right|} \int_{B_{r} \backslash U}\left[\left(v-C_{0} r^{\alpha}\right)^{+}+C_{0} r^{\alpha}\right]^{s} d x\right)^{\frac{p}{s}} \\
& \leq 2^{p}\left(\frac{1}{\left|B_{r}\right|} \int_{B_{r} \backslash U}\left(v-C_{0} r^{\alpha}\right)^{s}+\left(C_{0} r^{\alpha}\right)^{s} d x\right)^{\frac{p}{s}} \\
& \leq c\left(\frac{1}{\left|B_{r}\right|} \int_{B_{r}}\left(\left(v-C_{0} r^{\alpha}\right)^{+}\right)^{s} d x\right)^{\frac{p}{s}}+c C_{0}^{p} r^{\alpha p}
\end{aligned}
$$

for some constant $c>0$ depending only on $p$ and $s$. Here we use the notation $f^{+}=\max (f, 0)$. Since $u$ is continuous in $\mathbf{R}^{n} \backslash \bar{E}_{i}^{q}$ and $\bar{E}_{i}^{q}$ is $q$-thin at $x_{0}$, we infer from [15], Corollary 2.51, that

$$
\lim _{r \rightarrow 0} \frac{\left|B_{r} \backslash U\right|}{\left|B_{r}\right|}=0 .
$$

Hence for small radii $r$ we may apply Lemma 2.1 with $\kappa=\frac{1}{2}, p \leq s \leq \frac{n p}{n-p}$, and conclude that

$$
\left(\frac{1}{\left|B_{r}\right|} \int_{B_{r}}\left(\left(v-C_{0} r^{\alpha}\right)^{+}\right)^{s} d x\right)^{\frac{p}{s}} \leq c r^{p-n} \int_{B_{r}}|\nabla v|^{p} d x
$$

for all $r$ small enough. Here the constant $c$ depends only on $n, p$ and $s$. For $0<s<p$, we have

$$
\left(\frac{1}{\left|B_{r}\right|} \int_{B_{r}}\left(\left(v-C_{0} r^{\alpha}\right)^{+}\right)^{s} d x\right)^{\frac{1}{s}} \leq\left(\frac{1}{\left|B_{r}\right|} \int_{B_{r}}\left(\left(v-C_{0} r^{\alpha}\right)^{+}\right)^{p} d x\right)^{\frac{1}{p}}
$$

by the Hölder inequality. Hence the estimate (3.3) holds for all $0<s \leq \frac{n p}{n-p}$. It follows that

$$
\left(\frac{1}{\left|B_{r}\right|} \int_{B_{r}} v^{s} d x\right)^{\frac{p}{s}} \leq c C_{0}^{p} r^{\alpha p}+c r^{p-n} \int_{B_{r}}|\nabla v|^{p} d x
$$

for some constant $c>0$ depending only on $n, p$ and $s$. Since $|\nabla v| \leq|\nabla u|$ a.e. in $\mathbf{R}^{n}$, we easily infer the assertion of the theorem in the case $1<p<n$.

The borderline case $p=n$ can be treated similarly by using some easy tricks. We only point out the differences in the arguments. We consider an arbitrary exponent $t$ with $q<t<n$ and denote

$$
E=\left\{x \in \mathbf{R}^{n}: \int_{0}^{1}\left(r^{t-n} \int_{B\left(x_{0}, r\right)}|\nabla u|^{t} d x\right)^{\frac{1}{p-1}} \frac{d r}{r}=\infty\right\} \cup \bigcap_{i=1}^{\infty} \bar{E}_{i}^{q} .
$$

For $s=\frac{n t}{n-t}$ we proceed as in the case $1<p<n$. Now we apply Lemma 2.1 for $p=t$, and the inequality (3.3) takes the form

$$
\begin{aligned}
\left(\frac{1}{\left|B_{r}\right|} \int_{B_{r}}\left(\left(v-C_{0} r^{\alpha}\right)^{+}\right)^{s} d x\right)^{\frac{n}{s}} & \leq\left(\frac{1}{\left|B_{r}\right|} \int_{B_{r}}\left(\left(v-C_{0} r^{\alpha}\right)^{+}\right)^{s} d x\right)^{\frac{t}{s}} \\
& \leq c r^{t-n} \int_{B_{r}}|\nabla v|^{t} d x
\end{aligned}
$$


Here the first inequality holds just because we are free to assume that the integral average on the left-hand side of (3.4) is less than 1 . The case $0<s<\frac{n t}{n-t}$ is treated by using the Hölder inequality.

Remark 3.1. Let $u \in W^{1, p}\left(\mathbf{R}^{n}\right), 1<p \leq n$, and let $s$ be as in Theorem 1.1. Then the integral condition

$$
\int_{0}^{1}\left(\frac{1}{\left|B\left(x_{0}, r\right)\right|} \int_{B\left(x_{0}, r\right)}\left|u-u\left(x_{0}\right)\right|^{s} d x\right)^{\frac{p}{s(p-1)}} \frac{d r}{r}<\infty
$$

implies the standard Lebesgue point property

$$
\lim _{r \rightarrow 0} \frac{1}{\left|B\left(x_{0}, r\right)\right|} \int_{B\left(x_{0}, r\right)}\left|u-u\left(x_{0}\right)\right|^{s} d x=0 .
$$

This is easily seen by using the fact that

$$
\int_{0}^{1}\left(\frac{1}{\left|B\left(x_{0}, r\right)\right|} \int_{B\left(x_{0}, r\right)}\left|u-u\left(x_{0}\right)\right|^{s} d x\right)^{\frac{p}{s(p-1)}} \frac{d r}{r}<\infty
$$

if and only if

$$
\sum_{i=1}^{\infty}\left(2^{n i} \int_{B\left(x_{0}, 2^{-i}\right)}\left|u-u\left(x_{0}\right)\right|^{s} d x\right)^{\frac{p}{s(p-1)}}<\infty
$$

\section{REFERENCES}

[1] D. R. Adams and L. I. Hedberg, Function spaces and potential theory, Grundlehren der mathematischen Wissenschaften, Band 314, Springer-Verlag, Berlin, Heidelberg, 1996. MR 97j:46024

[2] D. R. Adams and J. L. Lewis, Fine and quasiconnectedness in nonlinear potential theory, Ann. Inst. Fourier (Grenoble), 35.1 (1985), pp. 53-73. MR 86h:31009

[3] D. R. Adams and N. G. Meyers, Thinness and Wiener criteria for non-linear potentials, Indiana Univ. Math. J., 22 (1972/73), pp. 169-197. MR 47:5272

[4] L. C. Evans and R. F. Gariepy, Measure theory and fine properties of functions, CRC Press, Boca Raton, Florida, 1992. MR 93f:28001

[5] H. Federer and W. P. Ziemer, The Lebesgue set of a function whose distribution derivatives are p-th power summable, Indiana Univ. Math. J., 22 (1972/73), pp. 139-158. MR 55:8321

[6] P. Hajlasz and J. Kinnunen, Hölder quasicontinuity of Sobolev functions on metric spaces, Rev. Mat. Iberoamericana, 14 (1998), pp. 601-622. MR 2000e:46046

[7] L. I. Hedberg, Non-linear potentials and approximation in the mean by analytic functions, Math. Z., 129 (1972), pp. 299-319. MR 48:6430

[8] J. Heinonen, T. Kilpeläinen and O. Martio, Nonlinear potential theory of degenerate elliptic equations, The Clarendon Press, Oxford University Press, New York, 1993. MR 94e:31003

[9] J. Kinnunen and N. Shanmugalingam, Regularity of quasi-minimizers on metric spaces, Manuscripta Math., 105 (2001), pp. 401-423. MR 2002i:35054

[10] T. Kilpeläinen and J. Malý, Supersolutions to degenerate elliptic equations on quasi open sets, Comm. Partial Differential Equations, 17 (1992), pp. 371-405. MR 93g:31022

[11] T. Kilpeläinen and J. Malý, The Wiener test and potential estimates for quasilinear elliptic equations, Acta Math., 172 (1994), pp. 137-161. MR 95a:35050

[12] J. Malý, Hölder type quasicontinuity, Potential Analysis, 2 (1993), pp. 249-254. MR 94i:31007

[13] N. G. Meyers, Continuity properties of potentials, Duke Math. J., 42 (1975), pp. 157-166. MR 51:3477

[14] V. G. Maz'ya and V. P. Khavin, Nonlinear potential theory, Uspehi Mat. Nauk 27 (1972), pp. 67-138; Russian Math. Surveys, 27.6 (1972), pp. 71-148. MR 53:13610 
[15] J. Malý and W. P. Ziemer, Fine regularity of solutions of elliptic partial differential equations, Mathematical Surveys and Monographs, No. 51, Amer. Math. Soc., Providence, RI, 1997. MR 98h:35080

[16] W. P. Ziemer, Weakly differentiable functions, "Sobolev spaces and functions of bounded variation", Graduate Texts in Mathematics, No. 120, Springer-Verlag, New York, 1989. MR 91e:46046

Department of Mathematics, University of Joensud, P.O. Box 111, 80101 Joensuu, FINLAND

E-mail address: visa.latvala@joensuu.fi 